\title{
World Allergy Organization Grading System for Systemic Allergic Reactions: it Is Time to Speak the Same Language When it Comes to Allergic Reactions
}

Mario Sánchez-Borges, M.D. ${ }^{1, *}$

Ignacio Ansotegui, M.D., PhD. ${ }^{2}$ Linda Cox, M.D. ${ }^{3}$

\author{
Address \\ ${ }^{*}, 1$ Allergy and Clinical Immunology Department, Centro Médico Docente La Trin- \\ idad, and Clínica El Avila, 6a transversal Urb. Altamira, piso 8, consultorio 803, \\ Caracas, 1060, Venezuela \\ Email: sanchezbmario@gmail.com \\ ${ }^{2}$ Department of Allergy and Immunology, Hospital Quironsalud, Bizkaia, Bilbao, \\ Spain \\ ${ }^{3}$ Nova Southeastern University, Fort Lauderdale, FL, USA \\ Published online: 13 September 2019 \\ (C) Springer Nature Switzerland AG 2019
}

This article is part of the Topical Collection on Specific Immunotherapy

Keywords Anaphylaxis · Allergic reaction · Severity · Systemic allergic reaction

Abstract

Purpose of review To discuss current methods for grading severe allergic reactions (SARs) in view of the new WAO modified classification system.

Recent findings While there is not universally accepted system for classifying SAR severity, and due to the high degree of heterogeneity and difficulties for comparisons between the various methods, the new WAO proposal is simple, suitable for use in clinical practice and universally applicable for all allergic reactions, covering the full spectrum of possible grades of severity.

Summary There is a requirement for a simple and comprehensive system to classify systemic allergic reaction severity. The modification to WAO's classification of SARs to allergen immunotherapy recently proposed by Cox et al. constitutes an important contribution in the goal of obtaining useful and helpful information for all involved stakeholders. 


\section{Introduction}

The severity of an allergic reaction is highly variable, ranging from local symptoms to generalized manifestations, being anaphylactic shock the most extreme and severe picture. According to the World Allergy Organization (WAO), anaphylaxis is defined as "a serious, lifethreatening generalized or systemic hypersensitivity reaction" and "a serious allergic reaction that is rapid in onset and might cause death" [1]. Severity of a particular reaction is influenced by many factors, such as dose, individual threshold for reaction, route of exposure, allergen, age, comorbidities, and cofactors.

Since there are huge differences in clinical features and degree of systemic compromise, and due to the relevance of reporting reactions in enough detail in order to generate precise information useful for patients, clinicians, the pharmaceutical industry, and health authorities, investigators have proposed various approaches to assess the severity of allergic reactions. In
2013, the World Allergy Organization proposed a uniform grading system to classify systemic allergic reactions (SARs) to subcutaneous allergen immunotherapy, based in involved organ systems and reaction severity [2].

Recently, a modification of this system applicable to all SARs due to any cause was published [3•]. This modified system was proposed to be applicable to SARs from any cause and allows for classification of less severe SARs which could be underreported or overreported in clinical trials and surveillance studies. So far there is not a widely accepted uniform classification system for grading SARs due to other causes such as drugs, foods, or insect stings. In this paper, we review the recent advances in the classification of SARs highlighting the current difficulties faced by allergy specialists to ensure a universal, simple, and widely accepted protocol for these conditions.

\section{Grading severe allergic reactions}

There are several grading systems to classify SARs induced by different agents. However, none of them is currently used worldwide. The uniform classification for grading subcutaneous immunotherapy-associated SARs created by WAO in 2010 [2] indicates that symptoms/signs from only one organ system could meet the criteria to be identified as a SAR. It is a 5-grade system based on the organ system(s) involved and the severity of the reaction, developed to classify reactions in a clinical scenario in which the putative triggering agent is known as able to cause a SAR. The reaction grade is determined by the physician or health professional after the episode is over, taking into account all factors related to the SAR, including among others the temporal relationship of symptoms/signs and exposure to the potential causative agent, and medications administered before and/or during the reaction. This system has also been applied to SARs induced by sublingual immunotherapy (SLIT).

\section{Current grading classification systems}

Due to the high variability in how SARs are reported, it is difficult to compare the safety outcomes associated with different practice patterns and therapeutic interventions. In general, existing SAR grading systems do not consider the reaction as a whole entity. Then, it is pertinent to determine the reaction grade by clinical judgement after the episode has finished, allowing the consideration of all involved factors, for example, the effects of concomitant exercise. Currently there is considerable intergrade variability in how SARs are classified, with similar reactions being graded in both the least and most severe categories. 
For example, reactions with a more rapid onset are often assigned a higher grade, even if the symptoms and signs do not progress. Most tools are designed empirically for data collection in emergency rooms or intensive care units, clinical trials, or based consensus reports, theoretical reviews, position papers, or national guidelines.

A harmonized severity scoring system ideally needs to take into account the perceptions and needs of different interested parties (patients, health workers, physicians, food and pharmaceutical industry, and public health authorities). An ideal scoring system for the severity of SARs should be based on easily recorded variables and should be applicable to all patient populations and to any acute allergic reaction. It would require to match the increase of involved organ systems with the increase in severity and to consider potentially lifethreatening manifestations (cardiovascular, neurological, and respiratory) as indicators of more severe reactions.

Scoring systems that have been used up to now were targeted to particular triggers such as foods [4-8], hymenoptera venoms [9-11], drugs [12, 13], and allergen immunotherapy [14].

These scoring systems classify severity in different grades valuing key symptoms and signs by using ordinal scales that are not equivalent across the different systems. Almost all of them are organ specific. Some of them utilize complex algorithms that include various elements such as dose, meeting 2 or more criteria, summation of symptoms, or number of involved organs and treatment plan. Some are targeted only for anaphylaxis and others are designed for a wider spectrum of reactions. None of these systems has been sufficiently validated.

As an example, Niggeman and Beyer proposed a 3-grade system to classify local and systemic reactions which was intended to overcome the disadvantages of current approaches [15]. However, it has been mentioned that this classification could result in considerable misrepresentation of the severity of a SAR $[3 \bullet]$.

\section{The need for a severity scoring system}

Among the reasons to establish an appropriate severity score system for SARs, the following have been mentioned: (1) A need to provide a summary of a reaction reported by patients or carers or within the context of a drug or food challenge or immunotherapy occurring in a clinical setting. (2) To contribute to determine the most appropriate emergency treatment. (3) To simplify into a small number of grades based on easily recognized symptoms and signs. (4) To have standardized patient monitoring and define patient cohorts in clinical trials.

Other confounding factors are the variable perceived severity and subjective interpretation of symptoms which depend on cofactors and circumstances surrounding patient's life. Allergic patients tend to underestimate or overestimate the severity of SARs, and frequently physicians underestimate the severity and treat SARs exclusively with antihistamines and corticosteroids, avoiding the use of epinephrine [16]. Another important issue to be taken into consideration is the lack of recognition or diagnosis of anaphylaxis by emergency room physicians with the consequent undertreatment [17]. In general, allergy 
specialists do not see the patient during an acute reaction and only assess severity retrospectively. Additional confounding factors are represented by patient's psychological comorbidities.

\section{Difficulties to design a uniform classification system for severe allergic reactions}

Among the triggering factors of SARs, the most common are foods, medications, insects, and physical factors. A number of obstacles for the design and implementation of a uniform classification for SARs have been considered.

1. A system covering all possible causes encounters with the impediment that the significance of symptoms/signs used to define an event as a SAR varies according to several circumstances, for example, allergen route of exposure and patient's ability to perceive and communicate his symptoms.

2. There is no reliable, evidence-based, gold standard for derivation and validation of SARs.

3. There is no reliability and validity due to sampling bias.

4. There is no harmonization in allergy nomenclature, although there is an important progress being made by the World Health Organization through

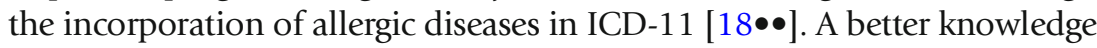
of disease mechanisms and endotype-driven approaches are lacking.

5. Symptoms and signs used to define a SAR are not specific to allergic reactions and can be present in idiosyncratic, immunologic, or other types of episodes.

6. SAR symptoms overlap with patient's anxiety.

7. Often the route of putative agent delivery can result in local reactions if the allergen is administered via the oral or sublingual route, or systemic if it is given parenterally.

8. Some grading systems include criteria that consider the treatment administered and the timing of response. Similar SAR grades of disparate severities could be assigned if there is a rapid resolution after epinephrine administration. Basing SAR grade severity on whether or not the physician or patient administers epinephrine can mislead treatment safety data and lead to a misuse of the term anaphylaxis.

9. There is the risk of underestimation of treatment safety if "anaphylaxis" and/ or epinephrine-treated reactions are the only SARs of concern and the only events prompting detailed reporting.

\section{Are there any comparisons between the different SAR classification systems available?}

Comparisons between the published systems are scarce. In a recent paper, Eller et al. compared 22 instruments using as the comparator Sampson's system [7]. The investigation included 2828 positive challenges to foods and drugs, and disclosed that there were significant 
correlations for mild reactions, whereas all instruments were good to identify food anaphylaxis. However, there were differences in severity between some instruments and the comparator system. Also 7 instruments were poor regarding drug anaphylaxis. Authors concluded that severity differed between the 23 instruments in both, food and drug allergy, and translation between scoring systems with 3 and 5 grades was difficult [19•].

\section{Modified SAR classification}

The accurate assessment and communication of potential severity of acute allergic reactions are important to patients, clinicians, researchers, food and pharmaceutical industry, and public health authorities. Many scoring systems to describe the severity of allergic reactions have been proposed, but there is considerable heterogeneity among them.

As mentioned, currently there is not a single standardized approach to quantify the severity of allergic reactions to all triggers. Recently, the European Academy of Allergy and Clinical Immunology recognized the need for the development of a harmonized system to quantify the severity of allergic reactions. These investigators proposed a 3-grade system in which grade 1 corresponds to isolated local reactions of skin and mucosa, grade 2 to reactions involving more distant sites (skin, upper airway, and/ or gastrointestinal tract), and grade 3 to potentially life-threatening reactions, cardiovascular, neurological, bronchial, and/or laryngeal. Patients would be assigned to a grade according to their most severe symptom/ sign $[20 \bullet]$.

Due to the multiple troubles for classifying SARs mentioned above, WAO has recently produced a modification of the 2010 WAO Grading System in order to implement a universal language for the classification of SARs due to any cause [3•]. This system allows symptoms/signs to be considered in the context of the entire reaction being the grade determined by a physician's clinical judgement. Grade severity is based on the organ system(s) involved and the severity of the SAR (Table 1).

Organ systems include the skin, upper and lower respiratory tracts, the conjunctiva, gastrointestinal, cardiovascular, and other symptoms. Single organ symptoms involving only one organ define a grade 1 reaction. Symptoms from two or more organs constitute a grade 2 or higher grade reaction. The presence of bronchospasm, gastrointestinal, or cardiovascular symptoms is grade 2 depending on its severity. Respiratory failure or hypotension, defined by the National Institute of Allergy and Infectious Diseases and Anaphylaxis Network Expert Panel criteria as a "reduced blood pressure after exposure to a known allergen for that subject (minutes to several hours)" [21], with or without loss of consciousness, is a grade 4 reaction (Table 1 ).

In the modified SAR Grading System, a grade 2 reaction is associated with symptom(s)/sign(s) from two or more organs from grade 1 . Mild lower airway, gastrointestinal symptoms, or uterine cramps are a grade 3, whereas more severe lower or upper airway or cardiovascular symptoms are grades 4 or 5 SAR. The final grade is determined by the physician 


\section{Table 1. Modified WAO Grading System for severe allergic reactions}

\section{Grades}

\section{Grade 1}

Symptom(s)/sign(s)

from one organ system

Cutaneous

-Urticaria and/or

erythema-warmth

and/or pruritus

other than localized

at injection site

and/or

-Lip tingling or

itching or

-Angioedema

(not laryngeal) or

Upper respiratory

-Nasal: sneezing,

rhinorrhea, pruritus

and/or congestion

and/or

-Throat clearing

(itchy throat) and/or

-Cough not related to

bronchospasm or

Conjunctival

-Erythema, pruritus,

tearing or

Other

-Nausea

-Metallic taste

\section{Grade 2}

Symptom(s)/sign(s)

From $\geq 2$ organ

symptoms listed

in grade 1

The final grade of the reaction is not determined until the event is over, regardless of the medication administered to treat the reaction. The final report should include the first symptom(s)/sign(s) and the time of onset after the causative agent exposure and a suffix reflecting if and when epinephrine was or was not administered: $a, \leq 5 \mathrm{~min} ; b,>5 \min$ to $\leq 10 \mathrm{~min} ; c_{,}>10 \min$ to $\leq 20 \mathrm{~min} ; d,>20$ min; $z$, epinephrine not administered. Final report: grades 1-5; $a-d$, or $z$; first symptom(s)/sign(s); time of onset of first symptom(s)/sign(s)

Modified from: Cox L, Sánchez-Borges M, Lockey RF. World Allergy Organization systemic allergic reaction grading system: Is a modification needed? J Allergy Clin Immunol Pract 2017; 5: 58-62

when the event has resolved. Additional information should also be reported, for example, first symptoms/signs, time of onset, timing, and amount of epinephrine administered. To denote when epinephrine was injected, an alphabetical suffix is used as follows: $a, 5$ min or less; $b, 5$ to $10 \mathrm{~min} ; c$, 10 to $20 \mathrm{~min}$; $d$, more than $20 \mathrm{~min} ; z$, if epinephrine was not administered.

This is a simple system that uses few grades, suitable for use in clinical practice and universally applicable for all allergic reactions, covering the full spectrum of possible grades of severity. 


\section{Conclusion}

Since there is a requirement for a simple and comprehensive system to classify systemic allergic reaction severity, a number of proposals have been published. However, they have been used empirically by investigators and up to now, there is not a model that has been accepted by all interested parties. The modification to WAO's classification of SARs to allergen immunotherapy recently proposed by Cox et al. constitutes an important contribution in the goal of obtaining useful and helpful information for all involved stockholders.

\section{Compliance with ethical standards}

\section{Conflict of interest}

Ignacio Ansotegui reports personal fees from Mundipharma, Roxall, Sanofi, MSD, Faes Farma, Hikma, UCB, and Astra Zeneca outside the submitted work. Mario Sanchez-Borges and Linda Cox declare no conflicts of interest relevant to this manuscript.

Human and animal rights and informed consent

This article does not contain any studies with human or animal subjects performed by any of the authors.

\section{References and Recommended Reading}

Papers of particular interest, published recently, have been highlighted as:

- Of importance

$\bullet \quad$ Of major importance

1. Simons FER, Arduso LRF, Bilò MB, El-Gamal YM, Ledford DK, Ring SJ, Sánchez-Borges M, Senna GE, Sheikh A, Thong BH, for the World Allergy Organization. World Allergy Organization guidelines for the assessment and management of Anaphylaxis. World Allergy Organ J 2011; 4: 13-37.

2. Cox L, Larenas-Linnemann D, Lockey RF, Passalacqua G. Speaking the same language: the World Allergy Organization subcutaneous immunotherapy systemic reaction grading system. J Allergy Clin Immunol. 2010;125(3):569-574, 574.e1-574.e7.

3. $\quad$ Cox L, Sánchez-Borges M, Lockey RF. World Allergy Organization systemic allergic reaction grading system: is a modification needed? J Allergy Clin Immunol Pract. 2017;5:58-62.

Important because it is a simple and comprehensive system for grading SARs.

4. Hourihane JO, Kilburn SA, Dean P, Warner JOC. Clinical characteristics of peanut allergy. Clin Exp Allergy. 1997;27:634-9.

5. Ewan PW, Clark AT. Long-term prospective observational study of patients with peanut and nut allergy after participation in a management plan. Lancet. 2001;357:111-5.

6. Cianferoni A, Garrett JP, Naimi DR, Khullar K, Spergel JM. Predictive values for food challenge-induced severe reactions: development of a simple food challenge score. Isr Med Assoc J. 2012;14:24-8.

7. Sampson HA. Anaphylaxis and emergency treatment. Pediatrics. 2003;111(6Pt3):1601-8.

8. Hourihane JO, Grimshaw KE, Lewis SA, Briggs RA, Trewin JB, King RM, et al. Does severity of low-dose, double-blind, placebo-controlled food challenges reflect severity of allergic reactions to peanut in the community? Clin Exp Allergy. 2005;35:1227-33.

9. Lockey RF, Turkeltaub PC, Olive ES, Hubbard JM, Baird-Warren IA, Bukantz SC. The Hymenoptera venom study. III: safety of venom immunotherapy. J Allergy Clin Immunol. 1990;86:775-80.

10. Golden DB, Kwiterovich KA, Kagey-Sobotka A, Lichtenstein LM. J Discontinuing venom immunotherapy: extended observations. J Allergy Clin Immunol 1998; 101: 298-305. 
11. Reisman RE. Natural history of insect sting allergy: relationship of severity of symptoms of initial sting anaphylaxis to re-sting reactions. J Allergy Clin Immunol. 1992;90(3Pt1):335-9.

12. Ring J, Messmer K. Incidence and severity of anaphylactoid reactions to colloid volume substitutes. Lancet 1977;1:466-9.

13. Ring J. Anaphylactoid reactions to intravenous solutions used for volume substitution. Clin Rev Allergy. 1991;9:397-414.

14. Allergen immunotherapy: Mueller HL. Further experiences with severe allergic reactions to insect stings. New Engl J Med 1959; 261: 374-377.

15. Niggeman B, Beyer K. Time for a new grading system for allergic reactions? Allergy. 2016;71:135-6.

16. Jares EJ, Baena-Cagnani CE, Sánchez-Borges M, Ensina LF, Arias-Cruz A, Gómez M, et al. Latin America Drug Allergy Interest Group Allergy. Drug-induced anaphylaxis in Latin American countries. J Allergy Clin Immunol Pract. 2015;3(5):780-8.

17. Sánchez-Borges M, Capriles-Hulett A, CaballeroFonseca F. Common mistakes in the treatment of patients with anaphylaxis. Rev Alerg Mex. 2014;61:35762.

18.• Tanno LK, Demoly P. Joint Allergy Academies. How can the World Health Organization's International Classification of Diseases (ICD)-11 change the clinical management of anaphylaxis? Expert Rev Clin Immunol. 2018;14:783-6.

Of outstanding importance because this article highlights the current WHO accepted nomenclature for allergic diseases and anaphylaxis.
19.• Eller E, Muraro A, Dahl R, Gotthard Mortz C, BindslevJensen C. Assessing severity of anaphylaxis: a datadriven comparison of 23 instruments. Clin Transl Allergy. 2018;8:29.

Important because this is one of few published papers comparing SAR grading methods.

20. Muraro A, Fernandez-Rivas M, Beyer K, et al. The urgent need for a harmonized severity scoring system for acute allergic reactions. Allergy. 2018;73:1792-800.

Important because this proposal is produced by a task force of EAACI, a prestigious Allergy Academy.

21. Sampson HA, Munoz-Furlong A, Campbell RL, Adkinson NF Jr, Bock SA, Branum A, et al. Second symposium on the definition and management of anaphylaxis: summary report-second National Institute of Allergy and Infectious Diseases/Food Allergy and Anaphylaxis Network symposium. J Allergy Clin Immunol. 2006;117:391-7.

\section{Publisher's Note}

Springer Nature remains neutral with regard to jurisdictional claims in published maps and institutional affiliations. 